\title{
Cytogenetics in head and neck cancer
}

\author{
Amita Pandey · Anupam Mishra
}

\begin{abstract}
Cytogenetics or the study of chromosomes has been an important tool in oncology. It localizes the abnormality on a particular chromosome segment as such but, the molecular analysis on the other hand focuses the exact gene of interest. Hence both are complimentary. Classical cytogenetics in combination with recent molecular techniques has given rise to various molecular cytogenetic analytical techniqucs such as florescent in-situ hybridization (FISH), spectral karyotyping (SKY) and comparative genomic hybridization $(\mathrm{CGH})$. The role of telomeres and its concerned enzyme telomerase is important in carcinogenesis. This article summarizes the various cytogenetic techniques and presents an overall view of the importance of cytogenetcs in head and neck cancer.
\end{abstract}

Keywords Cytogenetics - FISH - SKY - CGH

\section{Introduction}

Cytogenetics or study of cromosomes is an important diagnostic and prognostic tool for head and neck squamous cell canecr (HNSCC). To specify the genetic abnormality resulting in cancer, cytogenetics and molecular analysis have shown to be complementary in defining the specific gene aberration. While cytogenetics examines the whole cell and thereby observing multiple changes, molecular analysis focuses on one particular gene of interest. Cytogenetics has been used to define the particular segement of chromosome of interest from where the molecular analysis proceeds to define the presence (and amount) of a particular gene of interest.

Cytogenetics has been helpful for hematological malignancies with circulating cells such as leukemia, but has encountered difficulty with solid tumours (e.g head and neck cancer) due to several reasons:

1. Obtaining the dividing cells from a solid tumour samples is difficult and hence it becomes necessary to grow the tumour in vitro for a certain period of time. Also the low mitotic rate and small tumour sample adds to the difficulty.

2. Chromosomal changes are far more complex and occur in terms of losses or gains rather than translocations.

3. Solid tumours appear to result from accumulation of more genetic changes than the leukemias and the changes are the admixture of (i) abnormal production of normal gene product, (ii) production of abnormal gene product, (iii) loss of gene product through inactivation by mutation, translocation or deletion of the gene.

\section{Classical Gytogenetics}

The first solid tumours to be studied through classical cytogenetics were Burkiti's lymphoma (translocation from 
Chromosome 8 to 14 or 22), retinoblastoma (loss of Chromosome 13) and Wilm's tumour (loss of Chromosome 11). The two-hit hypothesis of Knudson [1] tends to explain the inactivation or loss of tumour repressor genes as evident by retinoblastoma / Wilm's tumour model. On the other hand Vogelstein's hypothesis [2] of progressive accumulation of genetic aberrations, more appropriately reflects the solid tumours. Alteration of three to six genes is though to be necessary for tumour development. Mutations in additional genes result in increased cellular proliferation, disorganization, tumour invasion and metastasis [2]. Moreover Califano et al [3] observed increased frequency of tumour suppressor gene loss as a function of increasing histopathological stage. The genetic changes in HNSCC may be conceptualized as a series of evolutionary events that may have neutral, deleterious or advantageous effects on the proliferation of a clone of cells. The Darwinian concept of 'survival of fittest' may apply for those genetic events that help the cells to proliferate, vascularize and metastasize against the normal immune mechanisms. The dividing cells either from culture or tissue sample are treated with colchicine for arresting the cell division and those cells with metaphase arrest are selected because the chromosomes are most prominent in metaphase. The chromosomes are then stained with Giemsa stain and 2 sets of data are obtained:

1. Identification of regions of chromosomes frequently deleted (possible sites of tumour suppressor genes).

2. Identification of chromosomal bands frequently involved in rearrangements and this may signify the site of oncogene activation.

Several regions of genome are preferentially deleted, duplicated or involved in chromosomal translocation which is in agreement with Vogelstein's hypothesis [2]. The karyotype of HNSCC exhibit clonal alteration and the differences from cell to cell reflect the heterogeneity caused by cytogenetic evolution that leads to various cell sub-populations present in the primary tumour from which the culture cells are derived. The most frequent karyotype change in HNSCC is tetraploidization [4]. Frequent structural chromosome-alterations in HNSCC include deletions, translocations, isochromosomes and unidentified marker chromosomes. Uncommon structural changes include duplications, insertions, inversions, ring chromosomes, dicentric chromosomes, and endoreduplicated chromosomes. Isochromosomes for almost every chromosome has been observed in HNSCC. Homogeneously staining regions of chromosomes seen as pale grey segments without any banding pattern are thought to harbor amplified genes. Important deletions predictive of tumorigenesis, in the order of frequency involve 18q, 13p, 10p, 8p, 15p, 22p as well as duplication of $7 \mathrm{p}$. Many breakpoint clusters however occur in heterochromatic regions at centromeres and not in euchromatic regions. The most likely sites for Oncogene implication in HNSCC carcinogenesis are reported to be
$1 \mathrm{p}, 3 \mathrm{p}, 4 \mathrm{q}, 5 \mathrm{q}, 7 \mathrm{q}, 8 \mathrm{p}, 10 \mathrm{q}, 11 \mathrm{q}$, I8q, 20p. In all, the frequent cytogenetic alterations in HNSCC include gains of 3q, 8q, $9 \mathrm{q}, 20 \mathrm{q}, 7 \mathrm{p}, 11 \mathrm{q} 13, \& 5 \mathrm{p}$ and losses of $3 \mathrm{p}, 9 \mathrm{p}, 21 \mathrm{q}, 5 \mathrm{q}, 13 \mathrm{q}$, $18 \mathrm{q}, 18 \mathrm{p}$. Figure 1 depicts conventional cytogenetics in a case of alveolar rhabdomyosarcoma.

\section{Molecular Gytogenetic analysis}

The molecular cytogenetic analysis was introduced in late 1980s. This not only revolutionized the held of cytogenetics but specially the cancer-cytogenetics and particularly improved the solid tumour assessment. Various techniques evolved with increasing complexity of cytogenetic assessment:

A. Florescent in-situ hybridization (FISH): is the primary technique used for molecular cytogenetic characterization of chromosomes, and involves hybridization of one or more fluorescently labeled DNA probes to metaphase chromosomes or nuclei. The usual form of FISH involves hybrid-

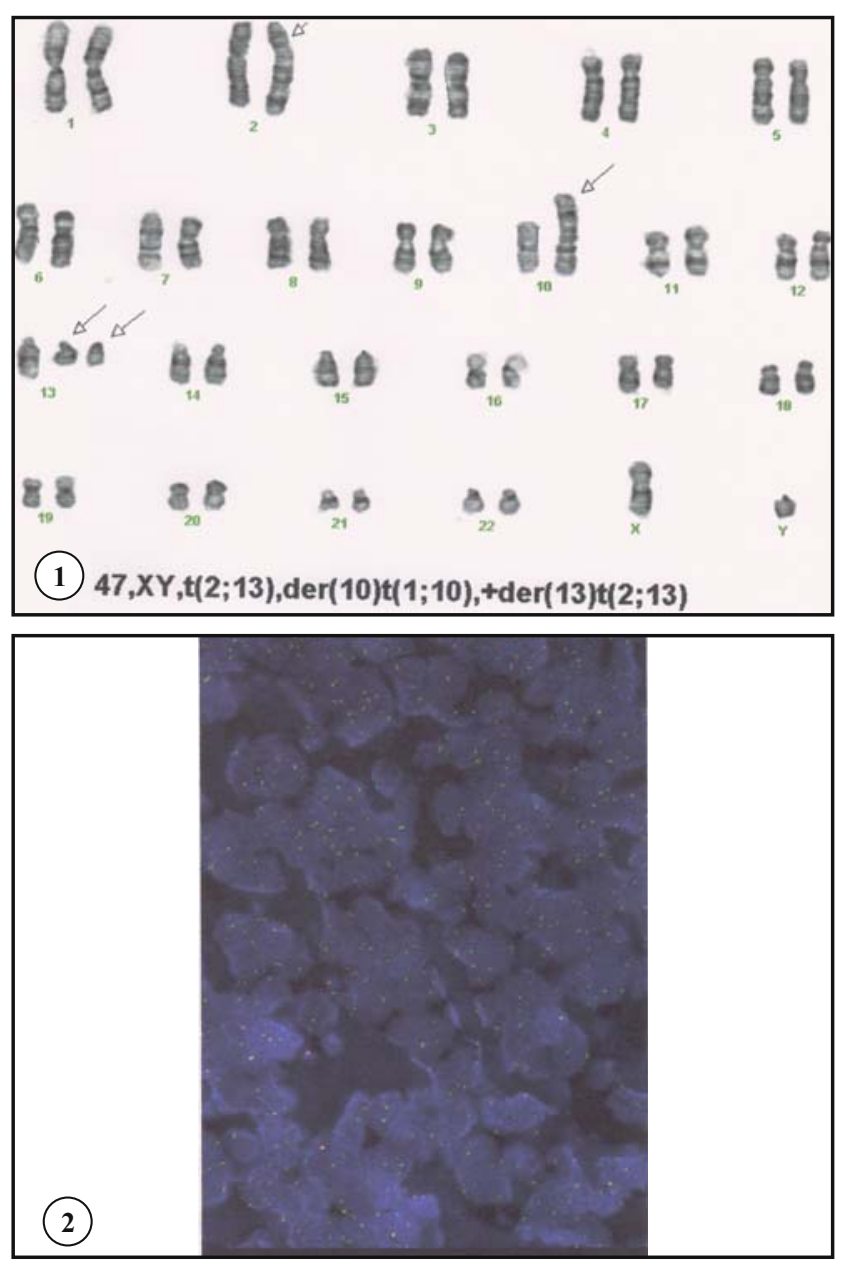

Fig. 1 Conventional cytogenetics in alveolar rhabdomyosarcoma Fig. 2 FISH using Vysis probe for FKHR in alveolar rhabdomyosarcoma 
izing such a probe to DNA segment / gene of interest and a different coloured probe to metaphase spreads from a tissue or cells of interest. Hence molecular cytogenetic analysis of targets defined by the DNA probe (eg centromeres by alphasatallite DNA, cosmid or bacterial-artificial-chromosome [BAC] clones of genes). For detection of chromosome or gene copy number or gene mapping can be carried out in context of deletions, amplifications or ploidy changes. The tissue preparation involves metaphase spreads or interphase nuclei from cultured cells, paraffin or frozen sections, touch preparations cytology brush preparations or dissociated tissue biopsy specimens. Optimally 200 cells are counted for this type of analysis. In telomere protocols this technique has been most commonly used. Figure 2 depicts FISH using Vysis probe for FKHR on a case of alveolar rhabdomyosarcoma.

B. Chromosome Painting: enables analysis of specific chromosomes or chromosome segments. Hence the identification of the origin of chromosomal segments involved in translocation and characterization of marker chromosome is possible, which otherwise is difficult by classical cytogenetic methods. The tissue preparation requires metaphase spreads from cultured tumour cells. The painting of 2 or more differentially labeled chromosomes in interphase nuclei provide little specific structural information owing to overlap of chromosomal domains. This can however be resolved by computerized optical sectioning with confocal microscopy.

C. Spectral Karyotyping (SKY) and multiplex FISH (MFJSH): These techniques are novel variations of chromosomal painting and are extremely useful for cancer cytogenetics. These distinguish 24 different human chromosomes by using a set of chromosome-specific DNA probes, each labeled with different array of florescent dyes and FISH hybridization to metaphase chromosomes. The CCD cameras with computer algorithms are used for detection and classification of rainbow of probes with SKY using Fourier spectroscopy to resolve multicoloured probes and M-FISH using multiple optical filters and image superimposition. The tissue preparation similarly involves metaphase spreads from cultured tumour cells.

D. Comparative Genomic Hybridization (CGH) developed by Kallioniemi et al [5] is a form of FISH that comprehensively screens the genome for gains and losses of DNA segments. It portrays an overall picture of genomic imbalances, eliminates the need for cell culture and thus overcomes the primary difficulty of karyotyping tumours with low mitotic index with consequent lack of metaphase chromosomes. However it has certain limitations: it cannot detect balanced chromosomal rearrangements viz. translocations and also it cannot suggest the pattern of loss in terms of chromosome rearrangements. Moreover CGH can only detect large genetic aberrations (gains of $2 \mathrm{mB}$ and losses of $10 \mathrm{mB}$ ) and a sufficient number of abnormal cells in the popula- tion ranging from $85 \%$ to $70 \%$ must be present to detect smallest and larger genetic abnormalities. Hence $\mathrm{CGH}$ analysis of tumour specimen is significantly affected by genetic heterogeneity encountered within the tumours and the frequent presence of normal cells. The tissue preparation involves normal male metaphase chromosomes and high molecular weight-DNA isolated from tumour cells. $\mathrm{CGH}$ involves isolation and labeling DNA from tumour cells in one colour while DNA from normal cells with another colour. The 2 DNAs are mixed and then hybridized to normal control metaphase chromosome spread. The chromosomes are identified by banding with DAPI stain, and the ratio of florescent dyes along the chromosomes is calculated to determine the location of chromosome gains, losses and amplifications. This procedure involves commercial imaging equipment along with computer system including proprietary software. CGH has overcome the inability of classical cytogenetic techniques to enable identification of the sources of some of the highly rearranged marker chromosomes (marker) in HNSCC. A major development in this technology was heralded by a technique labeled as matrix-CGH [6] by which the target DNA was immobilized onto glass slides and hybridized with biotinylated tumor DNA and digoxygenin-labeled reference DNA. These slides were labeled with two different florochromes, the ratios of which were analyzed by confocal microscopy. Through this technique it is possible to detect genomic gains and losses not detected by traditional CGH.

\section{Role of Telomeres}

Telomeres or the chromosomal caps contain six base pair sequence TTAGGG repeated many thousands of times. These serve at least three critical function: (1) To protect chromosome ends from enzymatic degradatio and abnormal fusion reactions; (2) To serve as a buffer zone to protect against the end-replication problem; (3) to serve as a scale for mitotic age (the divisional clock). Telomeric repeats are lost with each round of cell division, leading to a decrease in mean telomerase length. After a certain length, the short telomeric DNA sequences trigger entry into senescence pathway and ultimately lead to apoptosis. Only those rare cells which engage mechanisms that stabilize telomere length are capable of indefinite proliferation (or resulting in cancer). In $90 \%$ of human cancers, telomere stabilization is achieved by reactivation or up-regulation of telomerase. Telomerase is an RNA-protein complex that utilizes its RNA as a template for addition of TTAGGG repeats, thereby compensates losses due to end-replication problem. Hence for a cancer cell the telomerase activity has been reported to be much higher (at least 6 fold compared to their benign counterparts). Recent studies have focused the telomerase as a diagnostic, therapeutic and prognostic target. 
Molecular correlation of important cytogenetic abnormality in HNSEC

The comparision of the various types of molecular cytogenetic methods as shown in table 1 describes these tech- niques as being best complementary. Molecular genetic analyses have defined the probable genes corresponding to the structural chromosomal abnormality as detected through classical cytogenetics. The important structural abnormalities along with the corresponding genes are shown

Table 1 Comparison of cytogenetic techniques.

\begin{tabular}{|c|c|c|c|c|}
\hline & $\begin{array}{l}\text { Conventional } \\
\text { Cytogenetics nding) }\end{array}$ & FISH & SKY & CGLI \\
\hline $\begin{array}{l}\text { D } \\
\text { S } \\
\text { A } \\
\text { D } \\
\text { V } \\
\text { A } \\
\text { N } \\
\text { T } \\
\text { A } \\
\text { G } \\
\text { E } \\
\text { S }\end{array}$ & $\begin{array}{l}\text { Overview of changes ie } \\
\text { deletions, duplications, } \\
\text { inversions, \& } \\
\text { translocation. } \\
\text { Sensitivity of detection } \\
=5-10 \mathrm{mB}\end{array}$ & $\begin{array}{l}\text { Highly specific \& } \\
\text { sensitive } \\
\text { Mapping genes } \\
\text { Detects deletions, } \\
\text { inversions, } \\
\text { numerical changes, } \\
\text { \& translocations in } \\
\text { interphase nuclei \& } \\
\text { metaphase chromosomes } \\
\text { Sensitivity of detection } \\
=1 \text { kB } \\
\text { Highly focused, prior } \\
\text { knowledge of gene(s) } \\
\text { required }\end{array}$ & $\begin{array}{l}\text { Detection of complex } \\
\text { translocations } \\
\text { Defines the origin of } \\
\text { marker chromosome } \\
\text { Detects chromosome } \\
\text { boundaries in interphase } \\
\text { nucleus. }\end{array}$ & $\begin{array}{l}\text { Global view of gain, } \\
\text { loss \& amplification } \\
\text { Chromosomes from } \\
\text { samples not needed, but } \\
\text { only small amount of } \\
\text { DNA } \\
\text { Sensitivity of detection } \\
=5-10 \mathrm{mB}\end{array}$ \\
\hline
\end{tabular}

Table 2 Chromosomal abnormality with gene involvement

\begin{tabular}{lll}
\hline Structural chromosomal abnormality & Gene(s) implicated & Role of the gene \\
\hline 3p gain & AIS or p40/p73L & Over expressed \\
7p gain & EGFR & Over expressed \\
8q gain & MYC \& PTK2 & Over expressed \\
$11 q 13$ amplification & CCND1 & Over expressed \\
& EMSI, FGF3, FGF4 & NOT over expressed \\
20q gain & BCASI \& ZNF217 & Over expressed in breast tumour \\
$3 p$ loss & FHIT & Tumour suppressor gene \\
$8 p$ loss & Unknown 3 suppressor genes & \\
$9 p$ loss & P16/CDKN2A, & Tumour suppressor gene \\
& p1 5/CDKN2B, & \\
& p1 8/CDKH2C, & \\
$11 q$ loss & p1 9/CDKN2D & \\
$18 q$ loss & Unknown 2 suppressor genes & \\
& DCC, DPC4, MADR2, P15, SCCAI, & Tumour suppressor gene \\
\hline
\end{tabular}


in table 2. It is important to realize that none of the recent techniques can completely replace the traditional classical cytogenetics.

Hence the cytogenetic endpoints need to be incorporated for defining the differences in behaviour and response to therapy in HNSCC cases. Therefore the final aim of genetic analysis is to enhance survival by identifying useful predictors, recurrence risk-factors and treatment-response. This information in turn may be used to enhance earlier detection and more effective custom-made preventive / treatment strategies to minimize mortality and morbidity.

\section{References}

1. Knudson AG Jr. (1985) Heriditary cancer, oncogenes and antioncogenes. Cancer Research 45:437-443
2. Vogelstein B, Kinzler KW. (1993) The multistep nature of cancer. Trends in Genetics 9:138-141

3. Califano J, vander Riet P, Westra W, Nawroz Fl, Clayman G, Piantadosi S et al. (1996) Genetic progression model for head and neck cancer: implication for field cancerizatiori. Cancer Res 56(11):1152-1155

4. Shackney SE, Smith CA, Miller BW, Burholt DR, Murtha K, Giles HR. et al. (1989) Model for the genetic evolution of human solid tumours. Cancer Res 49(12):3344-3354

5. Kallioniemi A, Kallioniemi OP, Sudar D, Rutovitz D, Gray JW, Waldnaan F, Pinkel D. (1992) Comparative genomic hybridization for molecular cytogenetic analysis of solid tumors. Science 258(5083):818-821

6. Solinas- Toldo S, Lampel S, Stilgenbauer S, Nickolenko J, 13enner A, Dolmer H, Cremer T, Lichter P. (1997) Matrix based comparative genomic hybridization: biochips to screen for genomic imbalances. Genes Chromosomes Cancer 20(4): 399-407 